\title{
Long wavelength Infrared Detection, Bands Structure and effective mass in InAs/GaSb Nanostructure Superlattice
}

\author{
Merieme Benaadad ${ }^{1 *}$, Abdelhakim Nafidi ${ }^{1}$, Samir Melkoud ${ }^{l}$,Abderrazak Boutramine, ${ }^{1}$ \\ and Ali khalal ${ }^{l}$ \\ ${ }^{1}$ Laboratory of Condensed Matter Physics and Nanomaterials for Renewable energy, \\ University Ibn Zohr, 80000 Agadir, Morocco
}

\begin{abstract}
We have investigated in the bands structure and the effective mass, respectively, along the growth axis and in the plane of InAs $\left(\mathrm{d}_{1}=48.5 \AA\right) / \mathrm{GaSb}\left(\mathrm{d}_{2}=21.5 \AA\right)$ type II superlattice $(\mathrm{SL})$, performed in the envelop function formalism. We studied the semiconductor to semimetal transition and the evolutions of the optical band gap, $\mathrm{Eg}_{\mathrm{g}}(\Gamma)$, as a function of $\mathrm{d}_{1}$, the valence band offset $\Lambda$ and the temperature. In the range of 4.2-300 $\mathrm{K}$, the corresponding cutoff wavelength ranging from 7.9 to $12.6 \mu \mathrm{m}$, which demonstrates that this sample can be used as a long wavelength infrared detector. The position of the Fermi level, $\mathrm{E}_{\mathrm{F}}=512 \mathrm{meV}$, and the computed density of state indicates that this sample is a quasi-two-dimensional system and exhibits $\mathrm{n}$ type conductivity. Further, we calculated the transport scattering time and the velocity of electrons on the Fermi surface. These results were compared and discussed with the available data in the literature.
\end{abstract}

\section{Introduction}

The atmosphere absorbs weakly in the near and the long wave infrared (LWIR) wavelength range, which offer a high potential for long-range observation sensors. The detection of infrared radiations is very important for a wide variety of activities. LWIR photodetectors have a several specialized applications [1]. For instance, in astronomy to detect celestial objects, for night vision, in the military missile tracking, environmental sensing, in medical diagnosis, in meteorology and climatology, and, can be used also in remote sensing systems [2].

Despite the mercury cadmium telluride (MCT) consider as the most widely used infrared detector, it is not a perfect material system, due to the intrinsic shortcomings such as the poor uniformity of large area [3], the difficulty of alloy component adjustment, and contains toxic elements. Recently, the InAs/GaSb superlattice with a type-II band gap alignment, is a promising alternative to MCT technology in the LWIR detection in view of its various advantages [4], such as the adjustable band gap energy [5-6], the low Auger recombination process (due to the separation of heavy hole $\mathrm{hh}_{\mathrm{i}}$ and light hole lhi bands) [7], the directbandgap absorption, high absorption coefficient, high carrier lifetime and the greater stability

* Corresponding author: meryaadad@gmail.com 
due to strong bonds in the III-V materials [8], the large electron effective mass, in addition to the convenience of material growth and device preparation process [9]. The dark current density is an important indicator for measuring the performance of infrared detectors. This indicator can be reduced by proper the band engineering. Indeed, the fundamental band gap of InAs/GaSb superlattice can be designed with varying the thickness of the constituent's layers for strong broad band absorption.

The study of such semiconductor heterostructues properties requires the investigation of bands structure that describes all the subband behavior [10]. The commonly used methods is the $\mathrm{k} \cdot \mathrm{p}$ method, that has been successfully used to explain many physical phenomena. It is more efficient to provide the electronic structure [11].

In this paper, we have focused on the investigation of the electronic bands structure properties, energy subbands and carrier's effective mass in the growth direction and in-plan of $\operatorname{InAs}\left(\mathrm{d}_{1}=48.5\right) / \mathrm{GaSb}\left(\mathrm{d}_{2}=21.5 \AA\right)$, based on the envelope function formalism of k.p theory and the effective mass approximation [12]. We have discussed the effect of the well width on the band gap energy of SL, the carrier effective masses and the effect of temperature on the optoelectronic parameters. In addition to the density of state and Fermi level energy calculations.

\section{Computational details}

The general expression of dispersion relation of type-II SLs for light particles (electrons and light holes) subbands is given by [13-14]:

$$
\cos \left(k_{z} d\right)=\cos \left(k_{1} d_{1}\right) \cos \left(k_{2} d_{2}\right)-\frac{1}{2}\left[\left(\xi+\frac{1}{\xi}\right)+\frac{k_{p}^{2}}{4 k_{1} k_{2}}\left(r+\frac{1}{2}-2\right)\right] \sin \left(k_{1} d_{1}\right) \sin \left(k_{2} d_{2}\right)
$$

With $\mathrm{k}_{\mathrm{z}}$ and $\mathrm{k}_{\mathrm{p}}\left(\mathrm{k}_{\mathrm{x}}, \mathrm{k}_{\mathrm{y}}\right)$ the wave vector in the growth direction and in-plane of the superlattice respectively. We chose the origin of the energy $\mathrm{E}$ at the top of InAs valence band as shown in the Fig.1.

For a given energy, in each host material, the Kane model [15] gives the wave vector $\left(\mathrm{k}_{\mathrm{i}}^{2}+\mathrm{k}_{\mathrm{p}}{ }^{2}\right)$. Then, the energy $\mathrm{E}$ of the light particles (electron and light hole) is given by:

$$
\begin{cases}2 P_{1}^{2} \hbar^{2}\left(k_{1}^{2}+k_{p}^{2}\right)=3\left(E-\varepsilon_{1}\right) E & \text { for InAs } \\ 2 P_{2}^{2} \hbar^{2}\left(k_{2}^{2}+k_{p}^{2}\right)=3\left(E-\varepsilon_{2}-\Lambda\right)(E-\Lambda) & \text { for GaSb }\end{cases}
$$

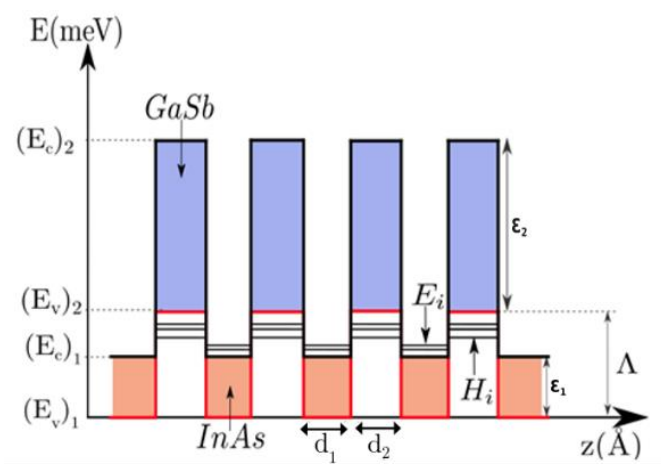

Fig. 1. Energy bands alignment in InAs/GaSb SL along the growth axis $z .\left(E_{c}\right)_{i},\left(E_{v}\right)_{i}$ and $\varepsilon_{i}$ where $i=1$, 2 are the conduction, the valence band edges and band gap of bulks, respectively. $\Delta \mathrm{E}_{\mathrm{v}}=\Lambda$ is the valence band offset. 
The expression of $\xi$ and $r$ in the Eq. (1) are:

$$
\xi=\frac{k_{1}}{k_{2}} r \quad r=\frac{E-\Lambda-\varepsilon_{2}}{E-\varepsilon_{1}}
$$

From the same equation (1), the type II superlattice heavy holes' mini-bands can be calculated with the following relations:

$$
\begin{cases}-\hbar^{2}\left(k_{1}^{2}+k_{p}^{2}\right)=2\left(m_{h h}^{*}\right)_{1} E & \text { for InAs } \\ -\hbar^{2}\left(k_{2}^{2}+k_{p}^{2}\right)=2\left(m_{h h}^{*}\right)_{2}(E-\Lambda) & \text { for GaSb }\end{cases}
$$

The valence band offset that we have adopted was $510 \mathrm{meV}$, that we calculated using the model given by [16]. $\mathrm{P}$ is the Kane matrix element whose values are $1.3610^{6} \mathrm{~J} / \mathrm{kg}$ for InAs and $1.4110^{6} \mathrm{~J} / \mathrm{kg}$ for GaSb [17]. Using these values of $\Lambda, \mathrm{P}$ and the input basic parameters of bulk materials the gap energy $\varepsilon_{\mathrm{i}}$ reported by [18] and the effective mass of heavy holes $\mathrm{m}_{\mathrm{HH}}^{*}\left(\mathrm{~m}_{0}\right)$ for each material $\operatorname{InAs}\left(0.41 \mathrm{~m}_{0}\right)$ and $\mathrm{GaSb}\left(0.40 \mathrm{~m}_{0}\right)$ given by [19], we have solved the general dispersion relation (Eq.1) in order to compute the bands structure for a fixed ratio $\mathrm{R}=\mathrm{d}_{1} / \mathrm{d}_{2}=2.26$ of this $\mathrm{SL}$ at $300 \mathrm{~K}$.

\section{Results and discussion}

We calculated the effect of InAs well's layer thickness, $d_{1}$, on subbands energies of light particles and heavy holes at the center $\Gamma\left(\mathrm{k}_{\mathrm{z}}=0\right)$ and the limit $\left(\mathrm{k}_{\mathrm{z}}=\pi / \mathrm{d}\right)$ of the first Brillouin zone (FBZ) as shown in Fig. 2. As we see, when $\mathrm{d}_{1}$ increases, $\mathrm{E}_{\mathrm{i}}$ decreases whereas $\mathrm{E}\left(\mathrm{HH}_{\mathrm{i}}\right)$ and $\mathrm{E}\left(\mathrm{lh}_{\mathrm{i}}\right)$ increases. Thus, the optical band gap energy $\mathrm{E}_{\mathrm{g}}(\Gamma)=\mathrm{E}_{1}(\Gamma)-\mathrm{H}_{1}(\Gamma)$ decreases until the crossover between $\mathrm{E}_{1}$ and $\mathrm{HH}_{1}$ that take place at the transition point $\mathrm{T}_{\mathrm{c}}\left(\mathrm{d}_{\mathrm{c}}=81 \AA \mathrm{A}_{\mathrm{c}}=\right.$ $462 \mathrm{meV}$ ) where $\mathrm{E}_{\mathrm{g}}=0 \mathrm{meV}$.

The conductivity of the system is converted from semiconductor ( $\mathrm{SC}$ with $\mathrm{E}_{\mathrm{g}}>0$ ) to Semimetal ( $\mathrm{SM}$ with $\mathrm{E}_{\mathrm{g}}$ small and negative). The electrons are moving from the top of $\mathrm{GaSb}$ into the bottom of the InAs [20]. Such charge transfer yields a rather high mobility in the

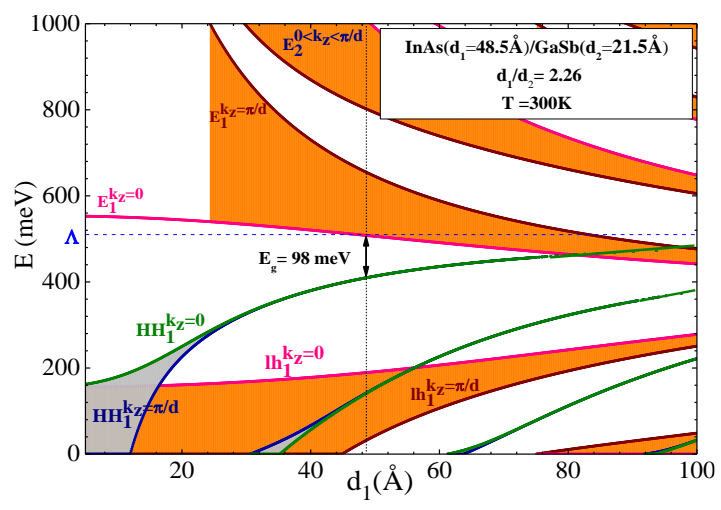

Fig. 2. Energy position and width of $\mathrm{E}_{\mathrm{i}}, \mathrm{HH}_{\mathrm{i}}$ and $\mathrm{lh}_{\mathrm{i}}$ subbands calculated at $300 \mathrm{~K}$, in the $\mathrm{FBZ}$ as a function of well thickness $d_{1}$. 


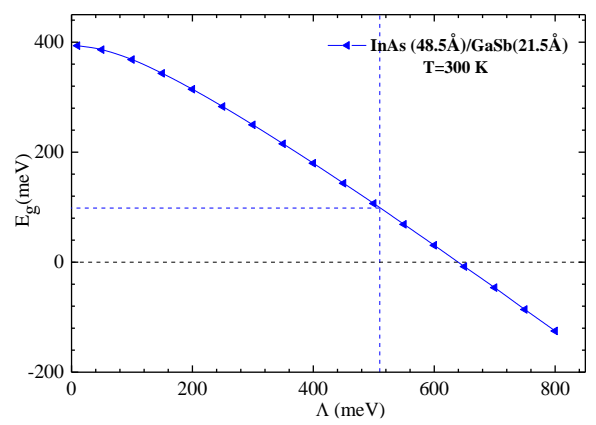

Fig. 3. Band gap, $E_{g}$, as a function of the valence band offset at $300 \mathrm{~K}$ in the investigated superlattice.

semimetallic samples since the electrons do not depend too much at low temperature of impurity scattering. This transfer process is similar to the well-known modulation doping in GaAs-GaA1As SL [21]. The semimetallic character and the semiconductor-semimetal transition of InAs/GaSb SL was studied experimentally well by transport and far infrared magneto- optical measurements by guldner et al.[22].

We used the measured electron carrier density $n$ as a function of the inverse of temperature by haugan et al.[23], we determine the experimental band gap in the intrinsic domain by plotting $\mathrm{nT}^{-3 / 2}$ as a function of $1000 / \mathrm{T}$. We found $\mathrm{E}_{\mathrm{g}}=95 \mathrm{meV}$, which is in agreement with $98 \mathrm{meV}$ calculated at $300 \mathrm{~K}$.

We illustrate in Fig. 3, the band gap $\mathrm{E}_{\mathrm{g}}$ as a function of band offset. The calculated $\mathrm{E}_{\mathrm{g}}$ decreases as the valence band offset $\Lambda$ increases. E $_{\mathrm{g}}$ decreases to zero at the transition semiconductor-semimetal and becomes negative accusing a semimetallic conduction beyond $\Lambda_{\mathrm{c}}=635.35 \mathrm{meV}$.

In Fig. 4, we plotted the evolution of coordinates of transition point for various temperature. The transition goes to a higher $\mathrm{d} 1 \mathrm{c}$ when the temperature decreases.

The effect of temperature on the band gap is presented in Fig. 5. At very low temperatures, the band gap is constant. Then, it decreases as the temperature increase from $188.6 \mathrm{meV}$ at $4.2 \mathrm{~K}$ to $98 \mathrm{meV}$ at $300 \mathrm{~K}$. Such behavior is due to the increased thermal energy and expansion of lattice constant which increase the amplitude of atomic vibrations and the interatomic spacing increase, then the potential seen by the electrons decreases, leading to a smaller band gap [24].

In order to determine the type of infrared detector of this sample, we calculated the cutoff wavelength using this formula:

$$
\lambda_{c}(\mu \mathrm{m})=\frac{1240}{E_{g}(m e V)}
$$

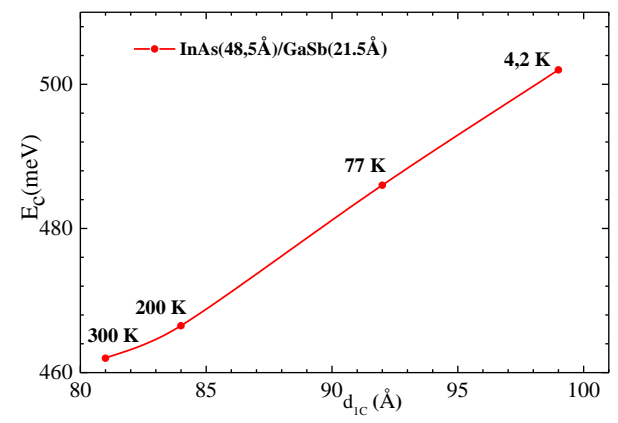

Figure 4: The coordinates of transition point at different temperature. 


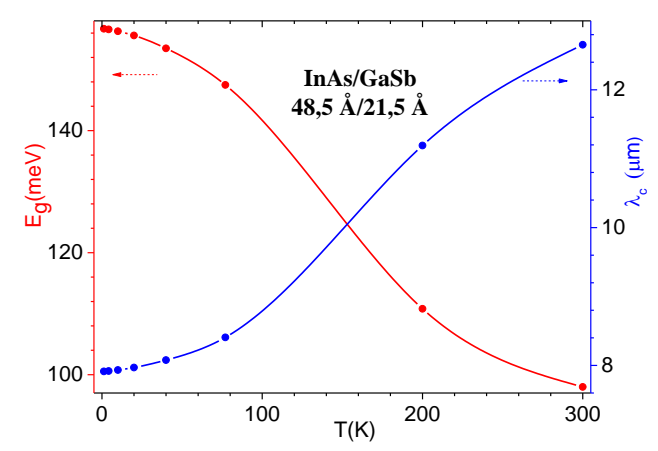

Fig.5. the variation of $E_{g}(T)$ and $\lambda_{c}(T)$ with temperature in the investigated SL.

As shown in Fig. 5, in the investigated temperature range of 1.2-300 K the corresponding cutoff wavelength is $7.9 \leq \lambda_{\mathrm{c}}(\mu \mathrm{m}) \leq 12.6$. Thus, this system can be employed for long wavelength infrared detection. Even, InAs/In $\mathrm{Ga}_{1-\mathrm{x}} \mathrm{Sb}$ SLs have been examined for infrared detector applications in the $1-19(\mu \mathrm{m})$ wavelength range for very long-wave infrared detection [7].

In Fig 6, we show the calculated SL bands structures along the growth direction $\mathrm{k}_{\mathrm{z}}$ and in plane $\mathrm{k}_{\mathrm{p}}$. We observe the dispersion curves of $\mathrm{E}_{1}$ and $\mathrm{E}_{2}$ bands along $\mathrm{k}_{\mathrm{z}}$, with a width $\left|E_{i}\left(k_{z}=\pi / d\right)-E_{i}\left(k_{z}=0\right)\right|$ of $E_{1}$ and $E_{2}$ of $138 \mathrm{meV}$ and $198 \mathrm{meV}$ respectively. The presence of these dispersion indicates a three dimensional electrons gas.

We calculated the carrier's effective masses using the dispersion of energy curves (Fig. 6 ), turns out to be a tensor with nine components, and its matrix elements along $\mathrm{i}$ and $\mathrm{j}$ directions given by Kittel [25] :

$$
\frac{1}{m_{i j}^{*}}=\frac{1}{\hbar^{2}} \frac{\partial^{2} E(k)}{\partial k_{i} \partial k_{j}}
$$

Figure. 6 shows that along $\mathrm{k}_{\mathrm{p}} \mathrm{m}^{*}{ }_{\mathrm{HH} 1}\left(\mathrm{k}_{\mathrm{p}}\right)=-0.4 \mathrm{~m}_{0}$. While $\mathrm{m}^{*}{ }_{\mathrm{h} 1}\left(\mathrm{k}_{\mathrm{p}}\right)$ decreases from -0.0208 $\mathrm{m}_{0}$ to $-0.0466 \mathrm{~m}_{0}, \mathrm{~m}_{\mathrm{E} 1}^{*}\left(\mathrm{k}_{\mathrm{p}}\right)$ diverge at inflection points $\mathrm{k}_{\mathrm{p}}=0.0185 \AA^{-1}$. Along $\mathrm{k}_{\mathrm{z}}, \mathrm{m}^{*}{ }_{\mathrm{E} 1}\left(\mathrm{k}_{\mathrm{z}}\right)$ and $\mathrm{m}^{*}{ }_{\mathrm{h} 1}\left(\mathrm{k}_{\mathrm{z}}\right)$ diverge also at inflection points $\mathrm{k}_{\mathrm{z}}=0.0267 \AA^{-1}$ and $\mathrm{k}_{\mathrm{z}}=0.0282 \AA^{-1}$ respectively. The dashed vertical line indicates the Fermi wave vector $\mathrm{k}_{\mathrm{F}}$. At the center $\Gamma$ of the first Brillouin zone the calculated mass of electrons is $\mathrm{m}_{\mathrm{E} 1}=0.0248 \mathrm{~m}_{0}$, these values are in the same order

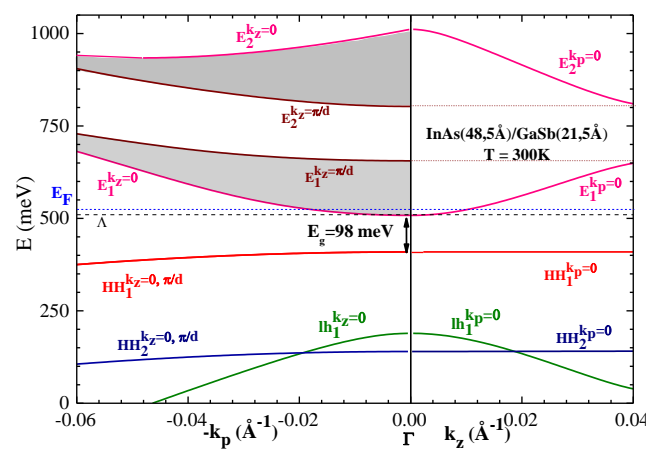

Fig.6. Band structures of the investigated SL along the $\mathrm{k}_{\mathrm{z}}$ and $\mathrm{k}_{\mathrm{p}}$ directions. 


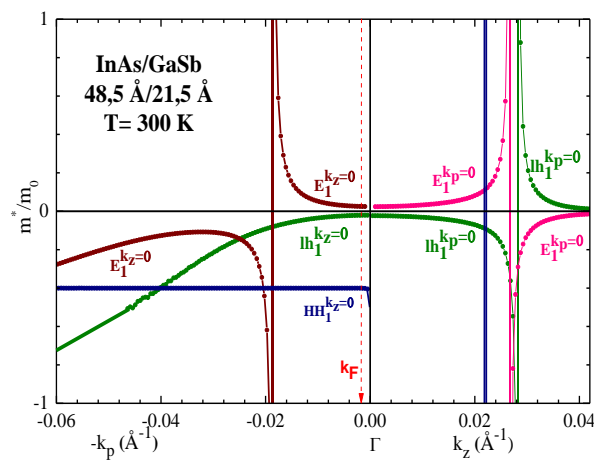

Fig. 7. Calculated carrier's effective mass along the growth direction and in plan of the SL.

than those determineted by [26] in a InAs-rich SL for a fixed ratio $\mathrm{R}=2,0.0270 \mathrm{~m}_{0}, 0.0295 \mathrm{~m}_{0}$ for a symmetric $\mathrm{SL}(\mathrm{R}=1)$ and $0.0480 \mathrm{~m}_{0}$ for $\mathrm{GaSb}$-rich $\mathrm{SL}(\mathrm{R}=0.5)$. Then, the electron effective mass is heaviest in the GaSb-rich SL then InAs-rich SL.

For the superlattice $i^{\text {th }}$ mini-band, with energy width $\Delta \mathrm{E}(\mathrm{i})=\mathrm{E}(\mathrm{i})_{\max }-\mathrm{E}(\mathrm{i})_{\min }$, the density of states (DOS) can be expressed as [27]:

$$
\rho_{\text {DOS }}^{(i)}(E)=\left\{\begin{array}{l}
\left(m^{*} / \pi^{2} \hbar^{2}\right) k_{z}(E) \text { for } E_{\min }^{(i)} \leq E \leq E_{\max }^{(i)} \\
0 \quad \text { otherwise }
\end{array}\right.
$$

The generalization of Eq. (6) required a sum over all mini-bands:

$$
\rho_{D O S}(E)=\sum_{i=1}^{n} \rho_{D O S}^{(i)}(E)
$$

In the absence of magnetic field, we calculated DOS of the two lowest conduction bands $\mathrm{E}_{\mathrm{i}}$ and the first valence subband $\mathrm{HH}_{1}$ and $\mathrm{lh}_{1}$ at $300 \mathrm{~K}$. As seen in Figure 8, the DOS is quantized in term of $\mathrm{m}^{*} / \pi \hbar^{2} \mathrm{~d}$. The observed dispersion in $\mathrm{E}_{\mathrm{i}}$ indicates the strong interaction between InAs wells.

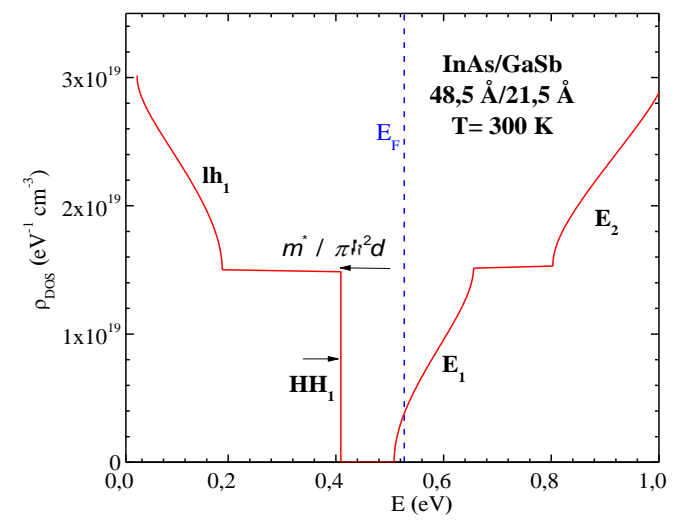

Fig.8. The SL DOS of the three subbands $\mathrm{lh}_{1}, \mathrm{HH}_{1}, \mathrm{E}_{1}$ and $\mathrm{E}_{2}$ at $300 \mathrm{~K}$. The vertical dashed line indicates the position of the Fermi level energy $\mathrm{E}_{\mathrm{F}}$. 


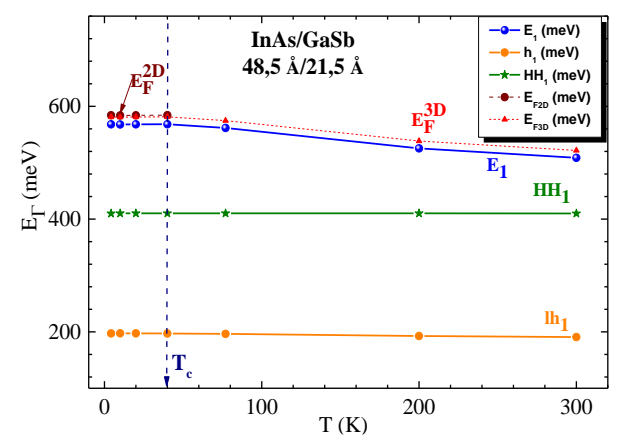

Fig.9. Energy of the first conduction $\left(\mathrm{E}_{1}\right)$, valence ( $\mathrm{hh}_{1}$ and $\mathrm{lh}_{1}$ ) bands, two and three dimensional Fermi level energy $\left(\mathrm{E}_{\mathrm{F}}\right)$ at different temperatures.

The Fermi level was calculated using the formula:

$$
\begin{aligned}
& E_{F}=E_{E 1}+\frac{\hbar^{2}\left(k_{F}^{i}\right)^{2}}{2 m_{E 1}^{*}} \text { with } \\
& k_{F}^{2 D}=(2 \pi n)^{1 / 2} \text { and } k_{F}^{3 D}=\left(3 \pi^{2} n\right)^{1 / 3}
\end{aligned}
$$

where $\mathrm{k}_{\mathrm{F}}^{2 \mathrm{D}}$ and $\mathrm{k}_{\mathrm{F}}^{3 \mathrm{D}}$ are the two dimensional and three dimensional Fermi wave vector respectively and $n$ is the measured concentration of electron charge in the $n(T)$ type sample [23]. The effective mass of electrons at the Fermi wave vector $\mathrm{k}_{\mathrm{F}}$ is $\mathrm{m}_{\mathrm{E} 1}\left(\mathrm{k}_{\mathrm{F}}\right)=0.0284 \mathrm{~m}_{0}$.

Using the formula 8, we calculate the Fermi level $\mathrm{E}_{\mathrm{F}}$, at the center $\Gamma$ of the first Brillouin zone, where the bands are parabolic as seen in Figure 6. In Figure $8 \mathrm{E}_{\mathrm{F}}$ is on the large $\mathrm{E}_{1}$ band which demonstrates n type and quasi-bidimensional (Q2D) conduction behavior. Although, the full first valence subband $\mathrm{HH}_{1}$ has a very narrow band width indicating 2D-like behavior.

In Figure 9, we plotted the energy bands of light $\left(\mathrm{lh}_{1}\right)$, heavy $\left(\mathrm{HH}_{1}\right)$ holes, and $\mathrm{E}_{1}$ electrons as a function of temperature which permits us to precise the dimensionality of system. The $\mathrm{E}\left(\mathrm{HH}_{1}\right)$ and $\mathrm{E}\left(\mathrm{lh}_{1}\right)$ still almost constant. Whereas The Fermi level $\mathrm{E}_{\mathrm{F}}(3 \mathrm{D})$ decreases with $\mathrm{E}_{1}$ when $\mathrm{T}$ increases. This indicates a three-dimensional electron gas behavior. While the energy of Fermi level $\mathrm{E}_{\mathrm{F}}(2 \mathrm{D})$ is constant.

For a $\mathrm{T}<\mathrm{T}_{\mathrm{c}}=42 \mathrm{~K}$, the electron gas behavior is Q2D while for $\mathrm{T} \geq \mathrm{T}_{\mathrm{c}}$ it's converted to 3D. We assist to an electronic conductivity transition Q2D-3D. Using the Hall mobility measured by [23] and our calculated effective mass of electrons $\mathrm{m}_{\mathrm{E} 1}$, we calculated the transport scattering time $\tau_{\mathrm{p}}=\mu_{\mathrm{H}} \mathrm{m}_{\mathrm{E} 1} / \mathrm{e}$, we found $0.27 \mathrm{ps}$ at $300 \mathrm{~K}$. This value of $\tau_{\mathrm{p}}$ can be compared to the quantum relaxation time $\tau_{\mathrm{q}}=0.5 \mathrm{ps}$ and $\tau_{\mathrm{p}}=2.34 \mathrm{ps}$ measured in AlGaN/GaN twodimensional electron gas [28]. We calculated also the velocity of electrons on the Fermi surface $\mathrm{v}_{\mathrm{F}}=\hbar \mathrm{k}_{\mathrm{F}} / \mathrm{m}_{\mathrm{EF}}^{*}$, it is about $3.9710^{4} \mathrm{~m} / \mathrm{s}$. This is a clear demonstration of faster collisions between electrons and phonon and high transport performances of this SL at high temperature.

\section{Conclusion}


We have performed a theoretical calculation based on the envelope function formalism in order to investigate the optoelectronic proprieties of $\operatorname{InAs}\left(\mathrm{d}_{1}=48.5 \AA\right) / \mathrm{GaSb}\left(\mathrm{d}_{2}=21.5 \AA\right)$ superlattice. We interpreted the effect of layer's thicknesses and temperature on the band gap and the corresponding cut-off wavelength. We calculate the intrinsic parameters of the SL such as the effective mass in plane and along the growth direction. The transition semiconductor to semimetal conductivity goes to higher $\mathrm{d}_{1 \mathrm{c}}$ when the temperature decreases. Our results show that this sample has n type tridimensional conduction behavior at $300 \mathrm{~K}$. The calculated cutoff wavelength indicates that this SL is a good candidate for the detection of long wave's infrared region. These results are guide for the design and engineering infrared detector.

\section{References}

[1] J. Liu, L. Xiao, Y. Liu, L. Cao, and Z. Shen, Chinese Phys. B 28, 2 (2019)

[2] A. Karim, J. Y. Andersson, A. Swedish, and I. C. T. Ab, IOP Conference Series: Materials Science and Engineering 51. 012001 (2013)

[3] A. Hood et al., Applied Physics Letters, 87 1-4 (2005)

[4] Y. Wei et al., Appl. Phys. Lett. 86, 1-3 (2005)

[5] G. J. Sullivan et al., J. Vac. Sci. Technol. B Microelectron. Nanom. Struct. 23, 1144 (2005)

[6] E. H. Aifer et al., Appl. Phys. Lett., 82, 4411-4413 (2003)

[7] C. H. Grein, P. M. Young, M. E. Flatté, and et al., J. Appl. Phys 78, 7143 ( 1995)

[8] A. Rogalski, M. Kopytko, and P. Martyniuk, Infrared Technol. Appl. XLIII 10177, 1017715 (2017)

[9] P. Martyniuk, J. Antoszewski, M. Martyniuk, L. Faraone, and A. Rogalski, Appl. Phys. Rev. 1, 4 (2014)

[10] C.Hamaguchi, Energy band structures of semiconductors. In Basic Semiconductor Physics, 1-63, Springer, Cham (2017)

[11] N. Luo, G. Liao, H. Q. Xu, N. Luo, G. Liao et al., AIP Advances 6, 125109 (2016)

[12] D. Barkissy, A. Nafidi, A. Boutramine, N. Benchtaber, A. Khalal, and T. El Gouti, Appl. Phys. A Mater. Sci. Process. 123, 1-7 (2017)

[13] G. Bastard, Phys. Rev. B 25, 7584 (1982)

[14] T. Andlauer and P. Vogl, Phys. Rev. B - Condens. Matter Mater. Phys. 80, 1-7 (2009)

[15] E. O. Kane, J. Phys. Chem. Solids 1, 249-261 (1957)

[16] K. Shim, Journal of Applied Physics, 114, 203703 (2013)

[17] J. Faist, Quantum cascade lasers, OUP Oxford. (2013)

[18] S. R. M. Levinshtein, Handbook Series on Semiconductor Parameters, (1996)

[19] M. S. Shur, Handbook series on semiconductor parameters. (1996)

[20] M.Altarelli, Physical review B 28, 842 (1983)

[21] L. L. Chang, N. J. Kawai, E. E. Mendez, Appl. Phys. 38, 30-32 (1981)

[22] L. L. Chang and L. Esaki, Applied Physics Letters 38, 30-32 (1980)

[23] H. J. Haugan et al., Journal of Applied Physics 125, 024505 (2019)

[24] H. Ünlü, Solid State Electron 35, 1343-1352 (1992)

[25] C. Kittel, Introduction to Solid State Physics. (2001)

[26] J. Imbert et al., Infrared Physics \& Technology 70, 81-86 (2014)

[27] H. . Cho, J. Vac. Sci. Technol. B Microelectron. Nanom. Struct. 7, (1989)

[28] A. Saxler, P. Debray, R. Perrin, S. Elhamri, W. C. Mitchel, and C. R. Elsass, MRS Internet J. Nitride Semicond. Res. 5, 619-625 (2020) 